\title{
Chatbots and Communication: The Growing Role of Artificial Intelligence in Addressing and Shaping Customer Needs
}

\section{Sae Bom Lee}

Kyung Hee University, Seoul, Korea

Objectives: This study examines the advantages of introducing chatbots and the different kinds of chatbot services in the e-commerce industry that are operating abroad. It also seeks to determine changes in future communication with companies and customers.

Methods: The main characteristics of e-commerce chatbots will be explored through four examples: the chatbots used by 1-800-Flowers, Sephora, PVR Cinemas, and Vainu.

Results: Sephora provides personalized services such as information delivery and recommendations through chatbots. 1-800-Flowers provides useful information to customers through interactive commerce services, as well as voice orders through Alexa for a convenient purchasing experience. PVR Cinemas supports purchasing and reservation functions to quickly solve customer problems, while Vainu has demonstrated that it can acquire customer information and use it for marketing by asking various questions to customers. Through these four cases, this study confirmed that product search, upselling, and marketing functions are the main advantages that can be obtained through communication with customers through chatbots in e-commerce transactions.

Conclusions: It was confirmed that chatbots contribute to improving the productivity and efficiency of existing business operations, and in particular, improving the convenience of customers' shopping, ordering, and payment experiences. In addition, as companies attempt to provide new shopping experiences to customers using chatbots, communication with customers through chatbots in the e-commerce field is expected to increase in the future.

Key Words: Chatbot, e-Commerce Industry, Business Communication, Personalized Interaction, Shopping Experience

\section{Introduction}

As artificial intelligence $(\mathrm{AI})$ is rapidly developing, it is permeat-

Received: Feb 28, 2020 Revised: Apr 18, 2020 Accepted: May 25, 2020 Corresponding author: Sae Bom Lee

Big Data Research Center, Kyung Hee University, 26, Kyungheedae-ro, Dongdaemun-Gu, Seoul 02447, Korea.

Tel: +82-2-961-0508, Fax: +82-504-476-3464, E-mail: spring@khu.ac.kr

This is an Open Access article distributed under the terms of the Creative Commons Attribution Non-Commercial License (http://creativecommons.org/licenses/ by-nc/4.0/) which permits unrestricted non-commercial use, distribution, and reproduction in any medium, provided the original work is properly cited.

Copyright $\odot 2020$ Korean Association for Business Communication. ing our lives without our awareness. Among them, the chatbot in particular is rapidly being applied in various fields, including customer relationship management, commerce, and communication. A chatbot refers to a robot that communicates with humans based on AI and involves an AI program that operates with a request and response structure (Park, 2017).

According to market forecasting agency Markets and Markets (2019), the chatbot market is expected to grow from USD 2.6 billion in 2019 to USD 9.4 billion by 2024, at a CAGR (compounded annual growth rate) of $29.7 \%$ during the forecast period. Gartner (2011) has already predicted that more than $85 \%$ of customer interactions will be managed without people by 2020 , 
and it is expected to become the number one AI consumer application in the future. In April 2016, the Facebook CEO, Mark Zuckerberg, released a chatbot API (application programming interface) at F8 (Facebook eight) developer conference, wherein he announced that "the future of Facebook is in messengers" (Lim, 2016). With the advancement of technologies such as AI, the increasing customer demand for self-service is driving companies to use chatbots. This is because 24/7 customer support is available through chatbots, which reduces operating costs, thereby enabling companies to achieve a competitive advantage by providing quick responses to customer inquiries (Markets and Markets, 2019).

Chatbots were introduced from the viewpoint of automating the processing of simple inquiries from customer centers. Previously, a chatbot used to be based on general rules and searches; presently, the chatbot supports a variety of non-faceto-face tasks such as delivery/return/cancellation, hospital reservation and interview processing, purchase-related product recommendations, and public service complaints. Chatbots are consequently emerging as indispensable in that they support non-face-to-face tasks involving communication between companies and customers in the "untact" (un+contact) era brought about by the coronavirus disease (COVID-19) pandemic. This study aims to examine the chatbots that have been growing in influence in various industries. With the progress in AI technology, advanced chatbots have begun to appear. Although AIbased chatbots have been introduced and are being used in service in Korea, we will focus on the overseas applications. This study explores the advantages of introducing chatbots and the different kinds of chatbot services operating abroad, in addition to determining potential changes in communication between companies and customers.

It will be necessary for future research and development to examine the current status of overseas chatbot development being used in the e-commerce field and to discuss ways to apply it to the Korean e-commerce field chatbot development. This study examines the development status of AI interactive chat- bots in the field of e-commerce and aims to provide practical help for domestic AI content production companies to produce electronic commerce interactive chatbots. Therefore, the research questions reflecting this purpose are as follows.

First, what are the principles of the development of AI-based interactive chatbots and the recent developments?

Second, what is the principle of an intelligent chatbot system based on AI technology?

Third, considering the types of chatbot services through overseas cases, what are the ways to use them for domestic chatbot development?

\section{Research on Chatbot}

The interactive chatbot based on AI technology uses the natural language processing technology (NLP) of a computer program to enable users to chat with the chatbot and solve various problems while the chatbot responds appropriately (Kompella, 2018). AI technologies used in chatbots can be classified into five groups as shown in Table 1 (Choi \& Nam, 2019).

The type of chatbot depends on an operation method. There are rule-based chatbots and machine learning-based chatbots. In addition, there are one-time Q\&A chatbots and continuous conversational chatbots according to the information exchange method. According to the answer generation method, it can be classified into a search model chatbot and a generation model chatbot (Kim, Shin, \& Kang, 2018). The following figure shows four types of chatbots. The rule-based chatbot in Figure 1 is relatively easy to implement, but it takes a long time to well define many rules. The better the rules are, the higher the quality of conversation service is possible. The machine learning-based chatbot in Figure 2 uses machine learning algorithms to understand natural language and generate or select appropriate answers. The NLP module plays the role of decomposing the natural language input by the user using various linguistic resources. On the other hand, the NLU module grasps the meaning of the decomposed natural language and delivers it to a generation

Table 1. Al technology used in a chatbot

\begin{tabular}{ll}
\hline Technology & \multicolumn{1}{c}{ Explanation } \\
\hline $\begin{array}{l}\text { Pattern recognition } \\
\text { Natural language processing }\end{array}$ & $\begin{array}{r}\text { Pattern recognition technology that can recognize and identify voices, texts, and figures. } \\
\text { automatic translation, and answering questions. }\end{array}$ \\
$\begin{array}{l}\text { Semantic web } \\
\text { Text mining }\end{array}$ & $\begin{array}{l}\text { Semantic web technology that allows us to understand and process relationships and meanings. } \\
\text { Context-aware computing }\end{array}$ \\
\hline
\end{tabular}

Note. Al, artificial intelligence.

Adapted from "The use of Al chatbot as an assistant tool for SW education," by Choi and Nam, 2019. CC-BY-NC. 
User input

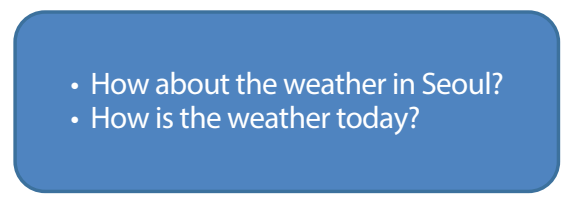

Interpretation rules

\begin{tabular}{|l|l|}
\hline \multicolumn{1}{|c|}{ Pattern } & Behavior sign \\
\hline Location weather & WEATHER \\
\hline DATE weather & WEATJER \\
\hline
\end{tabular}

Interpreting user input

- WEATHER SEOUL LOCATION

- WEATHER TODAY_DATE

Entity recognition

\begin{tabular}{|l|l|l|}
\hline Higher concept & Entity name & Expression \\
\hline LOCATION & SEOUL & Seoul \\
\hline DATE & TODAY & Today \\
\hline
\end{tabular}

Figure 1. Rule-based chatbot. Adapted from "Chabot technology introduction and case analysis," by Kim et al., 2018. Copyright 2018 by Korean Institute of Communications and Information Sciences. Reprinted with permission.

User input

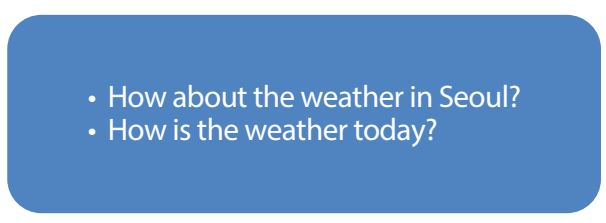

NLP

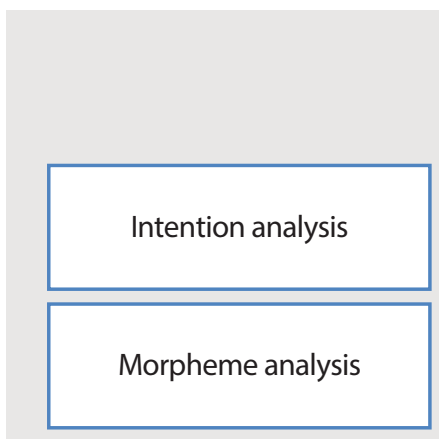

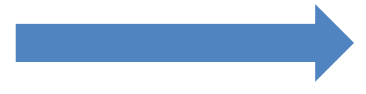

- WEATHER SEOUL_LOCATION

- WEATHER TODAY_DATE

Figure 2. Machine learning-based chatbot. Adapted from "Chabot technology introduction and case analysis," by Kim et al., 2018. Copyright 2018 by Korean Institute of Communications and Information Sciences. Reprinted with permission.

model to generate a refined answer. A significant amount of linguistic data is required because a high level of NLP technology is required. The one-time Q\&A chatbot in Figure 3 presents a set answer to frequently asked questions and is designed in a one-time Q\&A format. Figure 4 shows a chatbot system that operates according to a specific scenario for a specific topic (Kim et al., 2018).
Research on chatbot is ongoing in various fields. In the field of language education, we are studying how chatbots are used for language learning. The use of chatbots for learning English (Lee, 2018), the development of AI chatbots for elementary English teachers (Min, 2019), and language learning through chatbots (Fryer, Nakao, \& Thompson, 2019) are topics that have been studied. Research on the attitudes and behaviors of 
Who are the target audience for the QnA Maker tool? QnA Maker is primarily meant to provide a FAQ data source which you can query from your Bot/Application. Although developers will find this useful, content owners will especially benefit from this tool. QnA Maker is a completely no-code way of managing the content that powers your Bot/Application.

How do I login to the QnA Maker Portal? You can login with your Microsoft account.

Is the QnA Maker Service free?

Yes, currently the QnA Maker tool is tree to use. However, we do meter the usage per account. See the Subscription Keys section of the documentation for details.

My URLs have valid FAQ content, but the tool cannot extract them. Why not?

It's possible that the tool is not able to auto-extract $Q n A$ from valid FAQ URLs. In such cases, you have an option to copy-paste the QnA content in a txt and try ingesting it. Alternately, you can always editorially add content to your knowledge base.

What format does the tool expect the file content to be? We support two formats of files for ingestion.

\section{Chat}

Welcome You!

Sample QnA

Hello

Sample QnA

Question

\section{Answer}

Yes, currently the QnA Maker tool is free to use. However, we do meter the usage per account. See the Subscription Keys section of the documentation for details. Sample QnA

Type your message...

Figure 3. One-time Q\&A chatbot. Adapted from "Chabot technology introduction and case analysis," by Kim et al., 2018. Copyright 2018 by Korean Institute of Communications and Information Sciences. Reprinted with permission.

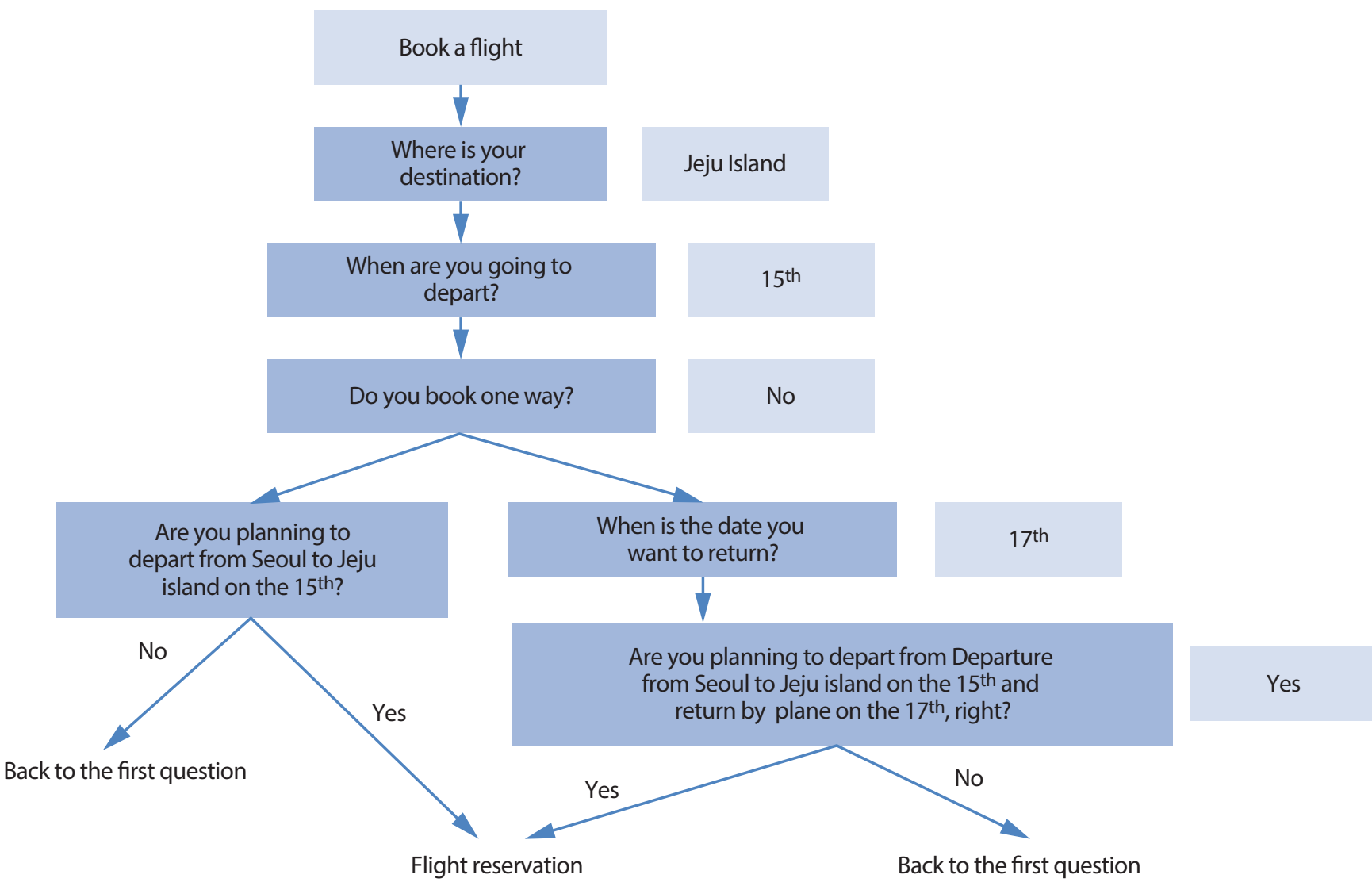

Figure 4. Continuous conversational chatbot-scenario-based conversation. Adapted from "Chabot technology introduction and case analysis," by Kim et al., 2018. Copyright 2018 by Korean Institute of Communications and Information Sciences. Reprinted with permission. 
consumers who use chatbots are also being conducted (Lee \& Park, 2019). This study introduces overseas chatbot cases in the field of e-commerce and compares them with Korean chatbot services. In this study, four cases are introduced.

Case studies are one of the most appropriate methods for exploring topics that have not been studied before (Yin, 1994), and this study attempted to introduce different services in the field of e-commerce by selecting multiple case study methodologies. The multi-case study method has the advantage of being able to explain the commonalities and differences between cases through comparative analysis by case. In this study, the study was conducted according to the existing study (Eisenhardt, 1991) that 4-10 cases are suitable when many representative cases cannot be found. Rather than presenting a large number of cases, the number of cases was selected with an emphasis on whether the number of cases and the number of newly acquired knowledge were proportional. Therefore, this study selected four representative cases and tried to select cases suitable for deriving the trend and advantage of the chatbot service using AI.

\section{Chatbot Services in E-commerce}

\section{1-800-Flowers: Ordering Flowers}

1-800-Flowers is an American florist and premium grocery retailer and distributor with annual sales of over USD 1.2 billion. It was also one of the first retailers to use interactive commerce over the Internet through chat or chatbot to sell directly to consumers. It is often considered an excellent example of a traditional company seeking to profit from the early phase of the newly introduced chatbot technology. 1-800-Flowers uses the Gwyn chatbot through Facebook Messenger. Its customers can order flowers and gifts through the chatbot and deliver them to anyone they want. The chatbot prompts customers to select items according to the type of event (birthday, romance, anniversary, etc.) and offers various gift options. They can buy flowers and receive delivery updates directly through the app. Previously, customers had to choose gifts directly from the e-commerce sites. Now, the site (https://www.1800flowers.com) can be easily navigated and customers can discover the right gifts and ideas according to the situation through a chatbot.

1-800-Flowers uses the Watson program (in partnership with IBM), an AI technology designed to transform the online customer experience with a smart virtual shopping assistant. 1-800-Flowers' chatbot uses these AI technologies to present customized product offers to customers. The advantage is that it can handle all kinds of questions, ranging from ambiguous to specific. Gwyn asks some follow-up questions to ensure that the right product offer is provided. For example, if a custom- er wants to find a gift for their mother, Gwyn enquires about opportunities, emotions, and the types of product she likes. In addition, the more customers interact with Gwyn, the more intuitive the shopping experience can be refined over time.

1-800-Flowers has partnered with Amazon to enable customers to order gifts through voice using Echo, Echo Dot, Amazon tap, or Alexa. It is a very convenient and visionary service when combined with AI technology, and can potentially attract tens of thousands of new customers. 1-800-Flowers offers one of the most complete e-commerce AI experiences, integrating numerous technologies across multiple channels and websites. Thus, it is dedicated to providing convenience and excellent service to end consumers (eTail, 2020). Figure 5 is an example of 1-800-Flowers' chatbot usage.

\section{PVR Cinemas: Booking Tickets for Movies or Events}

This chatbot is on a website and helps visitors quickly book movie tickets, view coupons, and leave feedback. Let us consider

People 1-800-Flowers.c... > Manage

\begin{tabular}{|l|l|l}
\hline & Gift Baskets & Get W \\
\hline ts & View Arrangements & Vi \\
\hline
\end{tabular}

\section{View Arrangements}
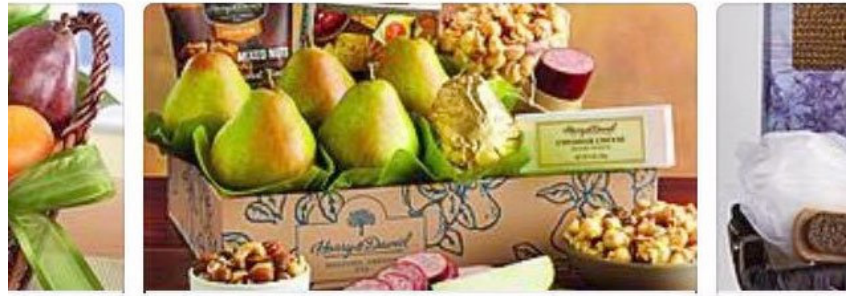

Gift

Harry and David ${ }^{\circledR}$ Fruit \&

Dena Snack Gift Boxes

Baske

$\$ 69.99$

$\$ 74.9$ s

Select this

Figure 5. 1-800-Flowers chatbot. Adapted from 1-800-Flowers.com website (https://www.1800flowers.com). 2020. Copyright 2020 by 1-800-Flowers.com. 
the case of PVR Cinemas, which owns one of the largest cinema chains in India. Its chatbot helps users make buying decisions by allowing its visitors to watch trailers of the movies they want to watch. This means that in addition to providing support, chatbots can also help increase sales. This chatbot application is not limited to cinemas. Performers, sports teams, organizations, nonprofit organizations, and anyone who creates events can use the chatbot to seamlessly sell tickets to fans and audiences.

PVR Cinemas reckoned that most customers ask the same type of questions about their purchases before making a decision to purchase a movie ticket. The company determined that increasing customer support efforts to answer these repetitive questions wasted valuable time and resources. As a better solution, the company deployed a chatbot on its website and designed it to answer basic questions that salespeople receive regularly. These questions include the following: Which movies are playing today? Which seats are available? Can I get a refund for my ticket? By answering these questions, the chatbot can guide customers and solve problems. In addition, a chatbot can also help the customer take the action they want (Rindell, 2019). Figure 6 shows the PVR Cinemas chatbot.

\section{Sephora: Personalized Recommendation for Beauty Products}

Sephora is a global company with over 2,300 stores in 33 countries worldwide. It operates a beauty retail store where customers can directly experience and purchase various products by selecting various cosmetic brands from the beauty industry. Sephora provides various services to customers through its chatbots, which use Kik Messenger. Customers take a quiz through a chatbot, delivering some of their key details, based on which the chatbot recommends the customer's product. It also provides personalized beauty tips, product recommendations, and reviews. Like a real salesperson, the Sephora bot asks questions about taste in order to recommend the perfect product. Customers can purchase the products they like without leaving the messenger, through Sephora's adaptation of Kik Messenger.

Sephora attempted to connect more effectively with customers online using AI. Facebook has developed a chatbot called Sephora Virtual Artist that allows people to try out lipstick colors using selfie photos. It also provides useful information for customers who want to learn about makeup through video clips and tutorials. The core objective of the Sephora bot is personalized product recommendation and product ordering through the chatbot (Quo, 2019). Figure 7 shows a list of lip gloss products being recommended through the Sephora chatbot.

\section{Vainu: Data Analytics Service for Sales and Marketing}

Companies can add chatbots to their websites and apps to engage customers. These chatbots can ask relevant questions, which provides a more engaging platform for customers to submit their contact information. In other words, the chatbot itself can serve as a great lead generation tool. When deploying a chatbot on a website, using rich conversations through the chatbot can engage customers in lead generation. Vainu, a data analysis service, uses VainuBot to ask questions to the customers who visit their website. Visitors can quickly select the option that is most relevant to them. Vainu can collect more information about their customers by asking multiple-choice questions through its chatbots and responding with new questions based on the visitors' previous answers.

In particular, Vainu initiates a thoughtful conversation ask-

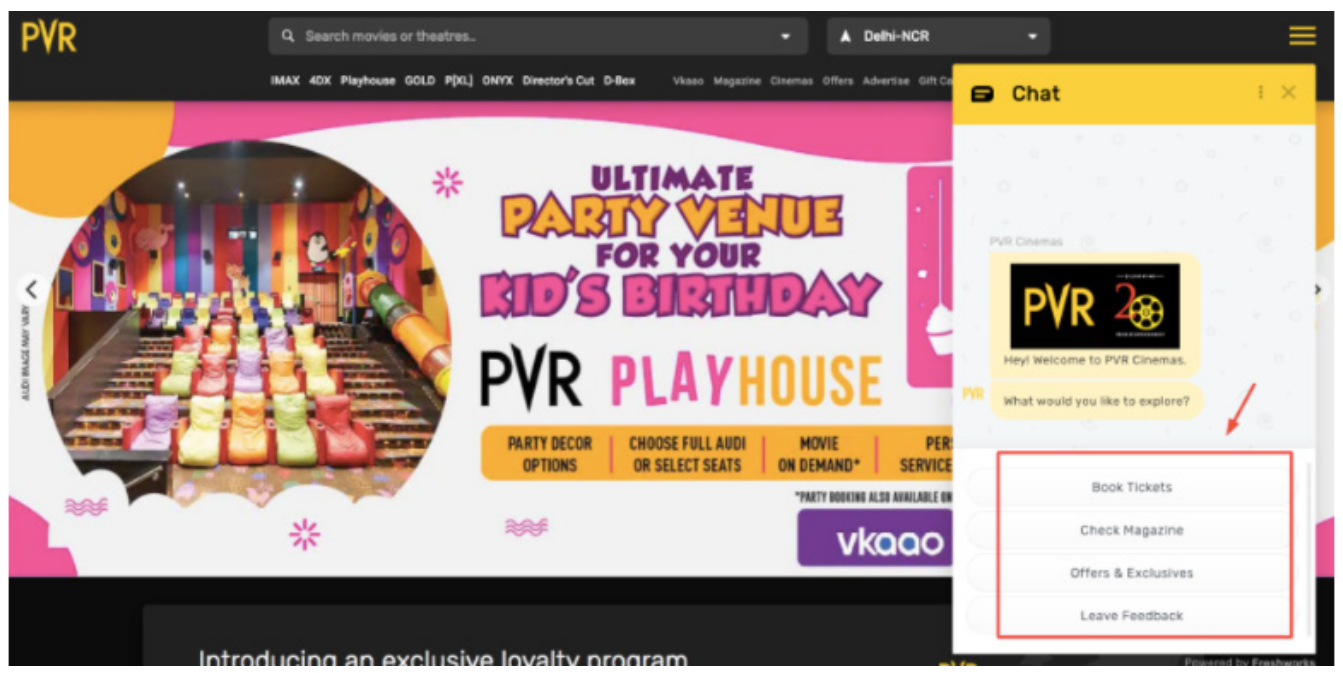

Figure 6. PVR Cinemas chatbot. Adapted from Sloboda Studio website (https://sloboda-studio.com). Copyright 2020 by Sloboda Studio. 


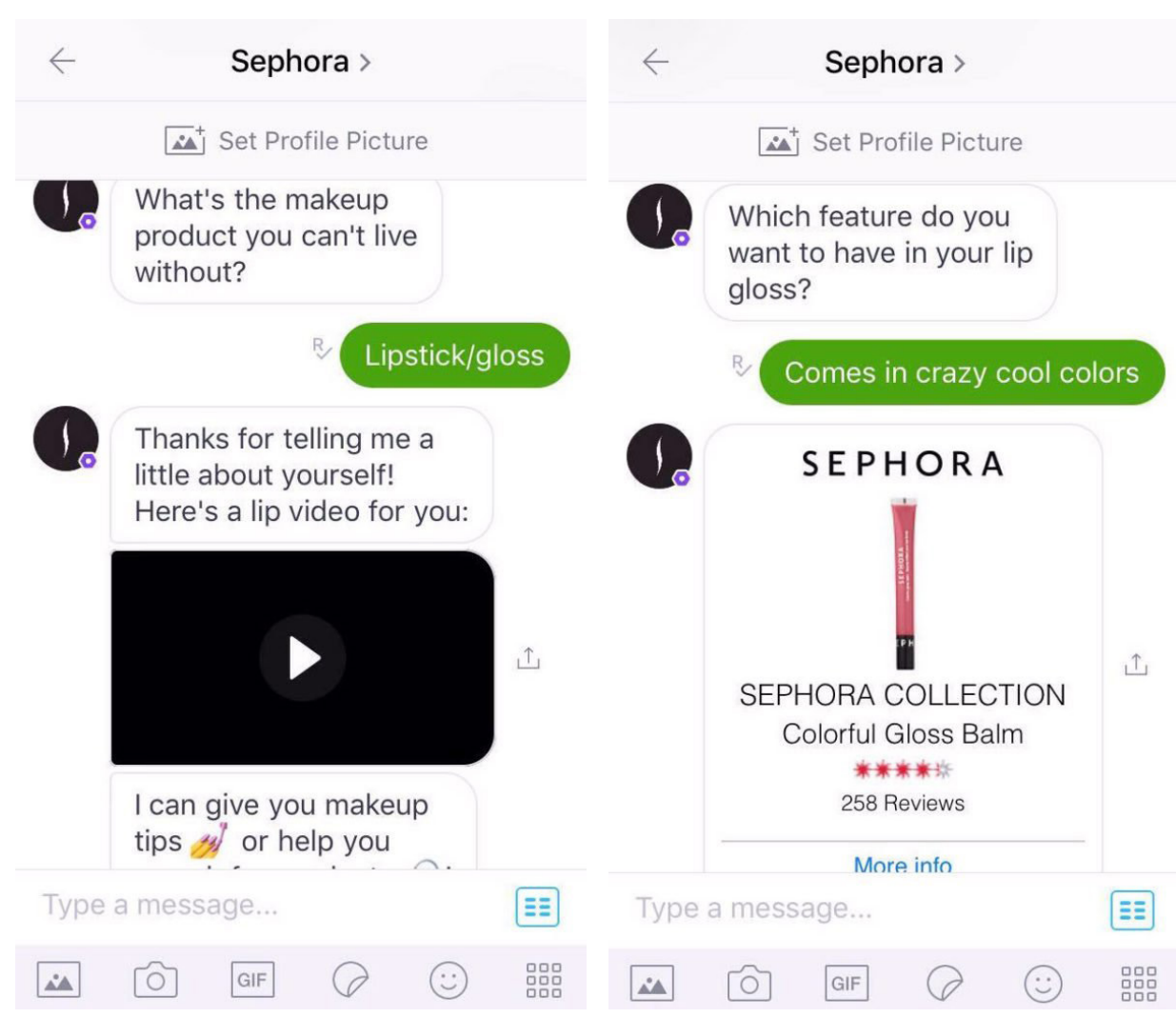

Figure 7. Sephora chatbot. Adapted from Sephora website (https://www.sephora.com). Copyright 2020 by Sephora.

ing visitors what they want to do through VainuBot to collect email addresses from website visitors. VainuBot initially asks its visitors what they want to do. When someone selects the "Check Content" option, they will be asked what topics they are interested in. Subsequently, depending on their selection, Vainu provides a link to a case study that the visitors can get if they want. The conversation is much better than the traditional format because the visitor gets involved in the conversation and receives appropriate responses to the questions. This dramatically increases the likelihood of a visitor submitting their email address in exchange for a case study because the chatbot promotes meaningful conversations. With VainuBot, Vainu makes visitors feel welcome on their website, listens to their needs and requirements, and asks if they are interested in useful content such as eBooks, email courses, or demos. By interacting with its visitors in this manner, they build trust and collect visitors' email addresses in exchange for providing useful content. This tactic allows businesses to send additional marketing emails that provide valuable content. Therefore, VainuBot generates leads on the website round the clock (Rindell, 2019). Figure 8 is an example of using VinueBot.

\section{Discussion}

Compared to other fields, chatbots have been around for quite some time in the field of e-commerce. When a shopper faced a problem or has a question, they used to call the company's call center, and probably had to wait an average of 11 minutes to talk to a real person. However, through a chatbot, shoppers can receive an immediate response and only need to wait about 45 seconds. Excluding customer service, which is the field that uses chatbots the most, the core functions of chatbots in the e-commerce field, as gleaned from the four cases, are as follows.

First, the assist in finding products and sharing delivery information. Instead of searching for a product on a website, customers can directly purchase the product they want by sending a simple message to a chatbot, such as the case of 1-800-Flowers. Customers can save the time spent on searching for a product on the website, as they do not have to go through multiple steps. Chatbots can process online transactions, send users to different pages, and accept payments within the bot itself, instead of elongating the payment process. In addition, if customers want to know when the purchased product will be shipped, the chatbots can access real-time data to keep customers informed about where their products are located. 
VainuBot

$$
\text { August } 16
$$

Would you like to improve your sales and marketing figures with the help of company data?

I guess the answer is yes! Is there something particular in your mind that $x$

brought you to our site today?

We need more sales

We need more/better prospects

Using on company data in sales

Using company data in marketing

Write a message

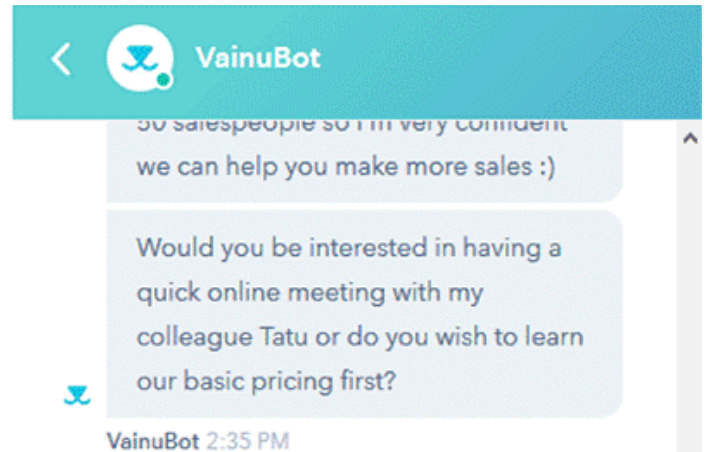

Price first!

2:35 PM

Could you share you email address with me at this point so in case we disconnect I can get back to you with

our pricing?

VainuBot 2:35 PM

Write a message

Figure 8. Lead generation by VainuBot. Adapted from GetJenny website (https://www.getjenny.com). Copyright 2020 by GetJenny.

Second, it can also upsell to customers. AI chatbots for e-commerce can identify products and services that customers are interested in. Hence, they can recommend high-quality products that will serve customers better.

Third, it performs a marketing function. Chatbots can collect valuable customer information through questions. Companies are able to achieve clearer advertising effects through chatbots than email advertisements. Moreover, chatbots can push notifications of new product launches and offers according to customer preferences. They can be used to create targeted campaigns according to user preferences, thereby making personalized marketing possible through past conversations and interactions. Therefore, advertising through chatbots becomes a more economical and efficient approach to reach customers than conventional means.

Table 2 summarizes the characteristics of the four cases in- troduced in this study. The four cases are mostly machine learning-based chatbots using NLP technology. In Korea, various companies in the field of e-commerce have developed and used chatbots. SSG.com uses a chatbot to look at today's dominance and tarot, and in the case of LG Electronics, through a chatbot, it provides consulting services such as product search and recommendation. However, the problem with most chatbots introduced in Korea is that chatbots do not come up with answers appropriate to the customer's question, and in the end, they are led to an agent connection. In addition, since there are many rule-based chatbots and one-time Q\&A types, it is difficult to receive product orders and product recommendations through natural conversations with a machine learning-based chatbot like in overseas cases.

In the future, in order to communicate smoothly with customers through chatbots in the field of e-commerce, the pur-

Table 2. Chatbot type by case

\begin{tabular}{llll}
\hline Case & \multicolumn{1}{c}{ Chatbot type } & \multicolumn{1}{c}{ Use Al technology } & \multicolumn{1}{c}{ Characteristics } \\
\hline 1-800-flowers & Machine learning-based & Natural language processing & Purchase the desired product through the chatbot \\
PVR Cinemas & Machine learning-based & Natural language processing & Providing quick movie reservation service \\
Sephora & Machine learning-based chatbot & Natural language processing & Product recommendation for each customer \\
Vainu & Scenario-based conversation & Context-aware computing & $\begin{array}{l}\text { Collecting customer leads and performing marketing } \\
\text { functions }\end{array}$ \\
\hline
\end{tabular}

Note. Al, artificial intelligence. 
pose of the service should be clear about what tasks the chatbot will replace. In addition, as in the case of overseas, a chatbot equipped with various functions for personalized recommendation should be operated. Like chatbots that sell products, features that allow chatbots to ask users first questions or induce games to understand users' tastes through games should be installed.

\section{Conclusion}

This study examined the business value that companies can obtain by communicating with customers through chatbots, based on the four e-commerce cases that successfully used such chatbots. Sephora provides personalized services such as information delivery and recommendations through its chatbots, while 1-800-Flowers provides useful information to customers through interactive commerce services, as well as voice orders through Alexa for a convenient purchasing experience. PVR Cinemas uses its chatbot to support purchasing and reservation functions to quickly solve customer problems, while Vainu has demonstrated that its chatbots can acquire customer information and use it for marketing purposes by asking various questions to customers. In this study, we examined cases where a chatbot's capabilities were successfully used in the field of e-commerce. Through this paper, it was confirmed that chatbots contribute towards improving the productivity and efficiency of existing business operations, and in particular, improving the convenience of customers' shopping, ordering, and payment experiences. In addition, as companies seek to provide new shopping experiences to customers using chatbots, communication with customers through chatbots in e-commerce is expected to increase in the future. The limitation of this paper is that it derives business value based on a small number of cases. In the future, we intend to derive business value based on cases of chatbot services across various industries.

\section{References}

Choi, S. W., \& Nam, J. H. (2019). The use of AI chatbot as an assistant tool for SW education. Journal of the Korea Institute of Information and Communication Engineering, 23(12), 1693-1699.

Eisenhardt, K. M. (1991). Better stories and better constructs: The case for rigor and comparative logic. Academy of Management Review, 16(3), 620-627.

eTail. (2020, February 25). Here's how 1-800-flowers leverages AI to streamline the customer journey. Retrieved from https:/etaileast. wbresearch.com/1-800-flowers-customer-journey-strategywith-ai-ty-u

Fryer, L. K., Nakao, K., \& Thompson, A. (2019). Chatbot learning partners: Connecting learning experiences, interest and competence. Computers in Human Behavior, 93, 279-289.

Gartner. (2011). Gatner customer 360 summit 2011. Retrieved from https://www.gartner.com/imagesrv/summits/docs/na/ customer-360/C360_2011_brochure_FINAL.pdf

Kim, S. G., Shin, M. C., \& Kang, J. Y. (2018). Chabot technology introduction and case analysis. KICS Information \& Communication Magazine, 35(2), 21-28.

Kompella, R. (2018, February 9). Conversational AI chatbot-architecture overview. Retrieved from https://towardsdatascience.com Lee, D. H. (2018). A study for the development of an English learning chatbot system based on artificial intelligence. Secondary English Education, 11(1), 45-68.

Lee, M. K., \& Park, H. J. (2019). Exploring factors influencing usage intention of chatbot - chatbot in financial service. Journal of Korean Society for Quality Management, 47(4), 755-765.

Lim, H. S. (2016, April 13). Facebook artificial intelligence chatbot released. Retrieved from https://www.yna.co.kr/view/ AKR20160413006700091

Markets and Markets (2019, November 19). Chatbot market by component (solutions and services), usage (websites and contact centers), technology, deployment model, application (customer support and personal assistant), organization size, vertical, and region - Global forecast to 2024. Retrieved from https://www. marketsandmarkets.com/Market-Reports/smart-advisormarket-72302363.html

Min, D. G. (2019). A study on the development of artificial intelligence(AI) chatbot by pre-service student teachers. Primary English Education, 25(4), 169-190.

Park, H. G. (2017). Read me chatbot. Excellence Marketing for Customer, 51(5), 40-50.

Quo, M. (2019, October 11). 10 Ecommerce brands succeeding with chatbots. Retrieved from https://www.abetterlemonadestand.com/ ecommerce-chatbots/

Rindell, M. (2019, September 04). Chatbot use cases: 25 real-life examples. Retrieved from https://www.getjenny.com/blog/chatbotuse-cases-25-real-life-examples\#Why-A-Chatbot-ImprovesCustomer-Suppo

Yin, R. (1994). Case study research: Design and methods. Thousand Oaks, CA: Sage. 